Fecha de recepción: mayo 2020 Fecha de aceptación: junio 2020 Versión final: julio 2020

\section{La identidad del Diseñador Gráfico del siglo XXI y la exigencia de la responsabilidad social universitaria}

Flor de María Gómez Ordoñez ${ }^{(*)}$

y María Teresa Alejandra López Colín ${ }^{(*)}$

\begin{abstract}
Resumen: Actualmente las Instituciones de Educación Superior (IES) se encuentran inmersas en un constante juego dialéctico por la responsabilidad que tienen como instituciones formadoras de futuros profesionistas frente a las expectativas de la sociedad. El ámbito educativo del Diseño no es la excepción; los futuros diseñadores están inmersos en la inercia que promueven las universidades a través de sus programas de estudio, en donde se destaca la relación que pronuncian las IES con el contexto social, es decir, se espera que los futuros profesionistas den respuesta a las necesidades de la sociedad; sin embargo, esta premisa se diluye al figurarse como un discurso plausible, pero no real y se describe sólo como un recurso para legitimar los esfuerzos de las universidades dentro de la Sociedad Global; así, esta participación tiene el objetivo de analizar la identidad del diseñador, traducida como el perfil profesional, indicador que evalúa la eficacia del proceso educativo y es, en sí mismo, el recurso para desafiar un ámbito laboral, en donde la misión de las IES se confronta y la identidad del egresado dista de los principios de la Responsabilidad Social Universitaria (RSU), que busca el reconocimiento de sí mismo y del otro, para una mejor sociedad.
\end{abstract}

Palabras clave: Identidad - Diseño - Diseñador - Perfil - Universidad

[Resúmenes en inglés y portugués en las páginas 172 - 173]

(*) Diseñadora Gráfica por la Facultad de Arquitectura y Diseño [FAD], de la Universidad Autónoma del Estado de México [UAEMéx.]; Maestra en Diseño y Producción de Contenidos Multimedia, por la Universidad La Salle, en Barcelona, España; y estudios de doctorado en Multimedia Educativo, en la Universidad de Barcelona. Profesora Investigadora de Tiempo Completo FAD, UAEMéx. Integrante del Cuerpo Académico Contexto Sociocultural del Diseño.

${ }^{(*)}$ Doctora en Educación por la Universidad de Ixtlahuaca CUI. Maestra en Administración por la Universidad de Ixtlahuaca, Diseñadora Gráfica por la Universidad Autónoma del Estado de México (UAEMÉX). Docente-investigadora de la escuela de diseño gráfico UI CUI, y profesora de asignatura en la licenciatura de Diseño Gráfico de la Facultad de Arquitectura y diseño de la UAEMéx. 


\section{Introducción}

En la obra Emilio de Rousseau (2000) se reconoce que el ser humano por naturaleza, desde sus primeros años de existencia cuenta con un espíritu abierto, inteligente y dispuesto a todo, que lo motiva a hacerse de un cúmulo de conocimientos que hace suyos; no busca conocer las cosas por su naturaleza, sino por las relaciones que le interesan y es por ello que bajo esa actitud y disposición sólo le hace falta conocer las virtudes de las relaciones sociales y lo que éstas le exigen como parte de una sociedad, en este momento, se hace necesaria la educación y la escuela, Savater al respecto menciona:

[...] educar es creer en la perfectibilidad humana, en la capacidad innata de aprender y en el deseo de saber que la anima, en que hay cosas (símbolos, técnicas, valores, memorias, hechos...) que pueden ser sabidos y que merecen serlo, en que los hombres podemos mejorarnos unos a otros por medio del conocimiento. (Savater, 1997, p.10)

Deval (2004) destaca la importancia de los objetivos que tiene la educación en la actualidad al contribuir al desarrollo psicológico y social del individuo, como el medio para integrarse a la sociedad, y ofrecerle los elementos esenciales de la cultura para la comprensión de la ciencia natural y humana; en este sentido, una institución educativa, con su estructura y sus actores, podrá contribuir a la transformación del individuo en un sujeto activo capaz de sociabilizar su conocimiento bajo una conducta, denominada como "prosocial":

Lo que la educación puede proporcionar son elementos para que esa adecuación a la práctica se realice con rapidez y facilidad. Pero para ello lo que se requieren son capacidades generales bien establecidas [...] Una persona capaz de pensar, de tomar decisiones, de buscar la información relevante que necesita, de relacionarse positivamente con los demás y cooperar con ellos... (Deval, 2004, p. 58)

Este mismo autor propone que los fines de la educación no pueden, ni deben ser eternos e independientes del contexto social, afirmación que dentro de la sociedad de la información y más aún, en la llamada sociedad del conocimiento, no podría ser la excepción, por lo que el desafío del sistema educativo de cualquier país tiene que ver con su adaptación a los medios, herramientas y lenguajes que plantea esta sociedad, reconociendo que las organizaciones educativas ya no son las únicas depositarias de la información y propiciadoras del conocimiento.

Bajo estas consideraciones, las Instituciones de Educación Superior (IES), se encuentran inmersas en una vorágine permeada por la cambiante complejidad social y la inmediatez generada por las siempre actuales Tecnologías de la Información y la Comunicación (TICs); en este panorama "Una de las mayores respuestas a la globalización consiste en construir y reconstruir la sociedad del saber y de la cultura; prolongar, y no reducir la formación" (Beck, 2008, pp. 255-256). Es así que diversas organizaciones mundiales, con diferenciados intereses, han especulado bajo el matiz neoliberal (Pérez, 2004), con relación a 
los propósitos necesarios y urgentes que debe alcanzar la educación, donde la mayoría de las veces, el conocimiento se ha concebido como una mercancía, el educando, susceptible de llegar a ser valorado como capital humano y el conocimiento, como indicador para la acreditación académica o para la legitimización profesional.

Por su parte, el Banco Mundial (BM), la Organización para la Cooperación y el Desarrollo Económicos (OCDE), y la Comisión Económica para América Latina y el Caribe (CEPAL), coinciden en que es un requisito de la Educación Superior cumplir

[...] con los ideales/objetivos/metas que esos mismos organismos proclaman, y que van ligados a la innovación, la integración de conocimientos, las economías, los actores e instituciones en distintos niveles, la competitividad, la viabilidad financiera y, junto con todo ello, la necesidad de que se muestre la utilidad de los conocimientos producidos. (Padierna, 2016, p. 118)

Desde esta premisa Fernández (2017), menciona que el reto y la necesidad de la educación superior ha sido vincular a sus actores educativos con los entornos locales, nacionales e internacionales, sin embargo, destaca que eso es posible a partir de generar capital humano capacitado para la conformación de sociedades más justas y economías más competitivas, función sustantiva que recae en las instituciones de educación superior. Cabe destacar el pronunciamiento de la Organización de las Naciones Unidas para la Educación, la Ciencia y la Cultura sobre la urgencia de integrar las competencias de la Educación para la Ciudadanía Mundial (UNESCO, 2015), para formar ciudadanos del mundo informados, dotados de espíritu crítico, socialmente conectados, éticos y comprometidos; mención que refiere a una clara visión del ciudadano integral que se conozca a sí mismo, en y para la sociedad, bajo una ética que permee la responsabildiad personal y social, en corcondancia con la ética planetaria (Morin, 2006), visión que se refiere a cultivar tres ámbitos de aprendizaje: cognitivo, socioemocional y conductual, para promover la paz, el bienestar, la prosperidad y la sostenibilidad en el ámbito social, desarrollando habilidades sociales para el saber ser y habilidades para la coordinación en conjunto como seres sociales.

Es pertinente destacar el panorama dicotómico que describe a la Educación Superior en México, la cual se suma a los principios y criterios establecidos de manera exógena y que vale la pena considerar, con el propósito de comprender el estado que guarda e incide sobre todo en el escenario del proceso de formación del diseñador gráfico del siglo XXI. En el contexto educativo mexicano, es la Asociación Nacional de Universidades e Instituciones de Educación Superior (ANUIES), el organismo no gubernamental que integra instituciones de educación superior y universidades de carácter público y privado; desde su fundación en el año de 1950, se ha comprometido en participar en la formulación de programas, planes y políticas nacionales, derivados de las políticas internacionales, y en definir las relaciones entre las agencias gubernamentales, las instituciones universitarias y la sociedad, (Ibarra, 2001, citado por Salas et al. 2019).

Una de las acciones representativas de la ANUIES ha sido la implementación de modelos de evaluación que tratan de reconfigurar el escenario educativo y mejorar la calidad educativa. Los planes y programas de estudio, así como el proceso enseñanza aprendizaje han 
sido los elementos considerados para dichas evaluaciones, bajo criterios calidad, excelencia y eficiencia, a la vez que los resultados se traducen en obtención de recursos económicos por parte del sistema educativo. Al respecto, Fernández menciona:

Estos tipos de evaluación ciertamente contribuyeron al ordenamiento de procesos sustantivos y lograron una política de rendición de cuentas. Sin embargo, de acuerdo con indicadores reportados por organismos como los Comités Interinstitucionales para la Evaluación de la Educación Superior, CIEES; el Consejo para la Acreditación de la Educación Superior, COPAES, y el Consejo Nacional de Ciencia y Tecnología, CONACYT, no se ha logrado establecer una mejoría en la calidad de la educación, a pesar de mostrar algunos avances debido a la transformación del trabajo académico. (2017, p. 189)

Dentro del ámbito de la educación superior del Diseño Gráfico, es el Consejo Mexicano para la Acreditación de Programas de Diseño A.C. (COMAPROD), reconocido por el Consejo para la Acreditación de la Educación Superior, A.C. (COPAES), el organismo que se constituye en el año 2003, a iniciativa de la Asociación Mexicana de Escuelas de Diseño Gráfico (ENCUADRE), con la misión de,

Generar una cultura universitaria de calidad en los programas de Diseño a través de la evaluación continua basada en marcos de referencia y parámetros de calidad que incentiven el desarrollo de la disciplina y difundir el conocimiento adquirido (COMAPROD, 2020, párr.1)

Es así que siguiendo los propósitos de la evaluación de la educación superior en el ámbito del Diseño Gráfico, COMAPROD, dentro del documento Marco de Referencia e Instrumento de Acreditación, establece que la evaluación es un recurso para la obtención de información sobre la calidad de las actividades académicas y de gestión, la cual "debe ser realizada en trescientos sesenta grados, con el fin de mostrar la diversidad de panorámicas que pueden observarse en cualquier programa de educación superior" (COMAPROD, 2017, p.5), teniendo como pregunta central ¿cómo debe enseñarse y aprenderse en el diseño?, cuestionamiento que se responde a partir del análisis del programa académico a evaluar, así como de las funciones sustantivas propias de una institución universitaria, en donde se integra la docencia, la investigación, la difusión y la vinculación.

Es en la función de la vinculación en donde se menciona que las instituciones de educación superior deben abrirse a otros actores de la sociedad, así como se reconocen tres tendencias en el ámbito del diseño: diseño como habilidad instrumental; el diseñador como intérprete o traductor de un lenguaje a otro; y el diseño como proceso de democratización en el uso de herramientas digitales. Asimismo, se destaca que el proceso formativo del diseñador gráfico debe contemplar

[...] investigación e integración de conocimientos; la correcta articulación entre teoría y práctica; la formación de sujetos críticos, capaces de incidir en 
las acciones de los usuarios; y la vinculación permanente entre la cultura y el desarrollo tecnológico. Este nuevo escenario exige una profesionalización real. Esto es igual a la formación de un profesional que otorgue servicios integrados y que sepa desplazarse por campos aparentemente distantes como los del branding y la cultura. Por último, dicho escenario demanda que la eficacia de los servicios de diseño sea comprobable al exterior de la propia profesión. (COMAPROD, 2017, p.9)

La situación que guarda el Diseño Gráfico dentro de las instituciones de educación superior, a través de sus programas de estudio, definen al diseño y al diseñador; de manera externa las políticas públicas marcan las pautas de calidad, excelencia y eficiencia. Por un lado, el contexto del diseño se autentifica desde los esfuerzos de cada institución de educación superior, pero por otro, como menciona Vallaeys y Álvarez (2019), éste se visualiza masificado y a expensas de un proceso de internacionalización de la calidad, sometido a clasificaciones e indicadores, característica sine qua non de cualquier programa que desea integrarse a la dinámica educativa global. Desde esta perspectiva se realiza la reflexión en torno a la educación superior como portadora del compromiso en la formación del diseñador, para descubrir el papel que juega en la responsabilidad social, al enfrentar al diseño con los requerimientos no solo desde la disciplina, sino desde el conglomerado social en el que se inscribe.

\section{Lo social en el diseño o el diseño en lo social}

La formación del diseñador gráfico, ha tenido un desarrollo gradual pero significativo, atrás quedó su antecesor, el aprendiz de las Artes Gráficas. Fue a partir de la Segunda Guerra Mundial cuando se reconoce al diseño de manera incipiente, con la modernidad se le exigen nuevos lenguajes, nuevas morfologías, en nuevos medios, lejos de la artesanía y de la producción en serie (Tapia, 2005b). Si bien Rodríguez menciona que el diseño de la modernidad se centra "en la forma que parte de un análisis de las necesidades y el contexto, para guiar un proceso racional que desemboca en la especificación de aquellos factores que determinan soluciones formales" (2010, p. 50), el factor moral del "buen diseño" estuvo considerado, al proponer soluciones a las necesidades de la sociedad, para la mejora de la calidad de vida a partir de la sencillez y la practicidad.

En su devenir el diseño y en particular el diseño gráfico, inicia su relación con otras ciencias y disciplinas, respondiendo a las demandas de instituciones públicas y privadas, antes que a las académicas. La academia se resignó a formar profesionistas que tuvieran un lugar en un contexto laboral, y con los nuevos medios que planteaba el desarrollo tecnológico. En los últimos años, el diseño gráfico ante un entorno siempre cambiante, desde la dimensión social, económica, ambiental y conductual, ha supuesto una nueva manera de abordar el diseño desde otras perspectivas como la sustentable, el diseño emocional, el diseño universal, el diseño social o el diseño centrado en el usuario y adopta conceptos que refieren 
más allá del diseño, como usabilidad o el mismo Design Thinking, circunstancias que lo hacen posicionarse de lleno en la era de la posmodernidad, es decir, un diseño impregnado de fragmentos de conocimiento, en un mundo complejo y con una multitud de medios.

Se reconoce de manera común que el Diseño Gráfico cumple con varias funciones y se realiza en diferentes contextos, con diferentes soportes y materiales. Sin embargo, hacia una aproximación del concepto se describe como una actividad representacional, simbólica y creativa, cuya especialidad consiste en determinar anticipadamente a su realización, las características finales de una pieza gráfica visual, para cumplir con una serie de requisitos definidos de antemano: funcionales, formales, estéticos y simbólicos (Belluccia, 2007), y satisfacer las necesidades de comunicación expresadas por alguien, para un propósito específico. A partir del reconocimiento del Diseño Gráfico como un tipo de comunicación es que el concepto se amplía, Frascara (1994) da pautas más allá de una visión moderna, al mencionar que en la práctica, se diseñan eventos, en donde el usuario interactúa con el diseño y se produce comunicación; este autor menciona que el objetivo del diseñador es el diseño de situaciones comunicacionales, esta aseveración coincide con Buchanan:

El diseño es el arte de darle forma a los argumentos sobre el mundo artificial o creado por el ser humano, argumentos que pueden ser llevados a las actividades concretas de la producción en cada una de las áreas, con resultados objetivos que al final son juzgados por los individuos, los grupos y la sociedad. (Buchanan, citado en Tapia, 2005, p. 77)

El Diseño Gráfico en la esfera social, en el mundo de las creencias y de las opiniones se convierte en mediador en la búsqueda de acuerdos sociales; y para modificar una creencia o mantenerla incluye a la persuasión, proponiendo a su público formas de pensar y de estar en el mundo y cómo actuar en éste (Rivera, 2007). Esto coincide con Belluccia (2007), quien a partir de su experiencia como diseñador menciona como requisito para legitimar un 'buen diseño' es necesario que el diseñador recurra a sus recuerdos, experiencias anteriores, su sensibilidad, sus conocimientos, sus pasiones, o su pasado, por ejemplo, pero también a las características del entorno social a donde va dirigida la pieza de diseño.

El desarrollo del Diseño Gráfico se destaca como un continuum, por lo que posicionarlo desde el ámbito comunicativo es limitarlo. Se destaca su potencialidad para traspasar el límite de la disciplina, hacia la interdisciplina y transdisciplina; Torres (2014), al abordar la perspectiva del diseño crítico, destaca que el diseño si bien está al servicio de la sociedad, no se le puede concebir como la disciplina que resuelve problemas, sino que los encuentra, es decir, lo que media es la actitud y los valores del propio diseñador al " [...] dar a conocer lo incuestionable, indagar en lo establecido, [...] cuestiona el sentido comúnmente aceptado sobre lo que es investigar puesto que desvela lo que no ha sido dicho" (p. 29), constituyéndose el diseño como una manera de crear significados, más allá de los mercados y los medios. Postura que no dista de la aseveración que ya había propuesto Papanek, al mencionar que el diseñador tiene la función de descubrir "un problema cuya existencia nadie había sospechado antes, lo delimita y seguidamente intenta resolverlo" (1997, p.141), con nuevas y mejores soluciones, acorde a la complejidad que le plantea cierta realidad. 
Al respecto, León y Vivanco (2009), destacan que el estudiante de diseño no puede estar ajeno a su realidad social, por lo que está obligado a conocer, interiorizar y conceptualizar, para ofrecer con sus propuestas de diseño una experiencia de integración social y cultural. Margolin y Margolin (2012), promueven el término diseño social, como la necesidad del estudiante de involucrarse no sólo en el área del diseño, sino también en áreas del conocimiento de la sociología, psicología y la política pública.

De esta manera el diseño se hace impensable fuera de la esfera humana y por ende, social, Gualdrón (2017) destaca al respecto, el First Things Manifiesto 2000, que inscribe dentro de su agenda el compromiso

[...] de practicar un diseño consciente que supone ser sensible al contexto, dar prioridad a la entidad humana, tratar el 'contenido' como algo que hacemos y no como algo que vendemos, concebir las diferencias culturales como valores positivos y no como obstáculos y centrarse más que en las cosas, para no inundar el mundo con artefactos carentes de sentido. (2017, p. 95)

En este mismo tenor la Asociación Profesional para el Diseño en los Estados Unidos Americanos (American Institute of Graphic Arts, AIGA), en el año 2015, brindó los resultados del estudio que tenían como propósito identificar los desafíos de las instituciones educativas, lo que permitió concluir acerca del "cambio de paradigma del diseño-forma al diseñocontexto-usuario y el diseño-arquitectura de información, privilegiando ética y contextos socioculturales en la práctica del diseño" (Gualdrón, 2017, p. 98). De acuerdo a Subirat y Badosa (2008), los diseñadores se encuentran en la encrucijada de apostar por un compromiso social, combativo del sistema o preocuparse por los aspectos formales, estéticos y mercantiles, es decir, constituirse como un talento en alquiler, dos polos que no necesariamente son opuestos, pero que generan la disyuntiva hacia dónde puede o debe ir el diseño.

\section{La Responsabilidad Social Universitaria (RSU)}

En este panorama y como ejercicio de reflexión vale la pena enfrentar el ser y hacer del diseño gráfico desde su ethos, que le exige cumplir con sus responsabilidades como actividad que trastoca la dimensión humana. Para Polo (2013) hablar del ethos remite al carácter para enfrentarse, por hábito con una misma realidad, Guzmán (2007) amplía el término desde la perspectiva filosófica y menciona que "un ethos es el espíritu que permea a un grupo social, un conjunto de actitudes y valores, de hábitos arraigados en el grupo" (p. 137). Bajo esta premisa, es preciso citar a Bauman (2004), en el sentido que en el tránsito mundial de la modernidad sólida a la modernidad líquida, implica estar preparados y ser asertivos para vivir la contingencia, la variedad, la ambigüedad, lo aleatorio y la idiosincrasia, desde el nuevo orden mundial. Es decir, el ethos, debe salir de su rigidez para apostar por la búsqueda de alternativas dentro de las políticas de vida. 
Papanek (1977) afirmaba que el diseño es la base de toda actividad humana y en cierto grado esta premisa ha dirigido a las instituciones que se han dedicado a formar diseñadores, desde esta perspectiva, las instituciones de educación superior y el proceso formativo no son entidades ajenas una de otra, para poder incidir en el ámbito social, "[...] debemos pensar en el hombre, sus recursos, su medio ambiente, y sus formas de pensar, planificar y manipularse a sí mismo y a su entorno, en cuanto un todo alinear, simultáneo, integrado y extenso" (Papanek, 1977, p. 255).

Esto último coincide con la motivación que se tuvo para generar el concepto Responsabilidad Social Universitaria (RSU) en América Latina, concebido en el año 2000 (Vallaeys, 2014), el cual tenía como propósito consolidar un concepto de Universidad Socialmente Responsable, basado en la gestión de los cuatro impactos que genera una institución de educación superior, a decir del autor, los que provienen de la misma organización; los del campus y su personal; los relacionados con la formación; y los que son producto de las relaciones con el entorno social.

La Responsabilidad Social Universitaria, en cierto grado deriva de la Responsabilidad Social Empresarial y fue vista en un primer momento como la posibilidad para describir y reconocer la intervención de cualquier institución universitaria en el ámbito social, desde la función sustantiva de la universidad dentro del área de la extensión (Tomassino y Cano, 2016), sin embargo, va más allá “[...] se pregunta por el tipo de profesionales, ciudadanos y personas que forma, y sobre la adecuada organización de la enseñanza para garantizar una formación socialmente responsable de sus estudiantes" (Zárate y García, 2014, p. 108). A la vez que representa una palanca de transformación en materia de educación superior, al demandar cambios no sólo en la posición y actitud de los actores, sino también en las políticas públicas, financieras y procesos de calidad, abonando responsabilidades adicionales a las instituciones de educación superior:

[...] remite a compromisos asumidos por las IES hacia sus integrantes (proporcionando una educación de calidad a sus estudiantes, incluyendo a grupos vulnerables, sensibilizando a los universitarios en cuestiones de orden moral y cívico y elevando la eficiencia de sus procesos de organización y gestión) y hacia el exterior (contribuyendo a resolver problemas de escala nacional o local, incidiendo en una convivencia armónica y participando de proyectos de desarrollo sostenible (Didou, 2015, p. 78)

La Responsabilidad Social Universitaria ha atravesado diferentes etapas que van más allá de las ayudas universitarias y los métodos solidarios de aprendizaje (extensión solidaria extracurricular), que se presentan frecuentemente en las instituciones de educación superior de manera parcial y de manera temporal. La responsabilidad social se define como, "la gestión integral y transversal de todos los impactos sociales y ambientales de las instituciones de educación superior, desde todos los procesos de formación, investigación, extensión y gestión organizacional, en miras a alcanzar los Objetivos del Desarrollo Sustentable en su ámbito social de incidencia" (Vallaeys, 2019, p. 13). Desde este planteamiento, la Unión de Responsabilidad Social Universitaria Latinoamericana (URSULA), autodefinida 
como el espacio de discusión crítica del rol de la educación superior en América Latina, ha propuesto el Modelo URSULA el cual busca transformar a la universidad en un laboratorio de la innovación social, a partir de trastocar el ethos, para llevar a la comunidad universitaria a situaciones originales y actuar en consecuencia, de esta manera propiciar el aprendizaje y el actuar significativo, en sí generar coherencia ética, de la institución y sus actores con respecto a lo que viven y en donde pueden incidir de manera real, para contribuir desde su especialidad en el entramado social, como un compromiso primigenio que deben visualizar las instituciones de educación superior (Figura Núm. 1).

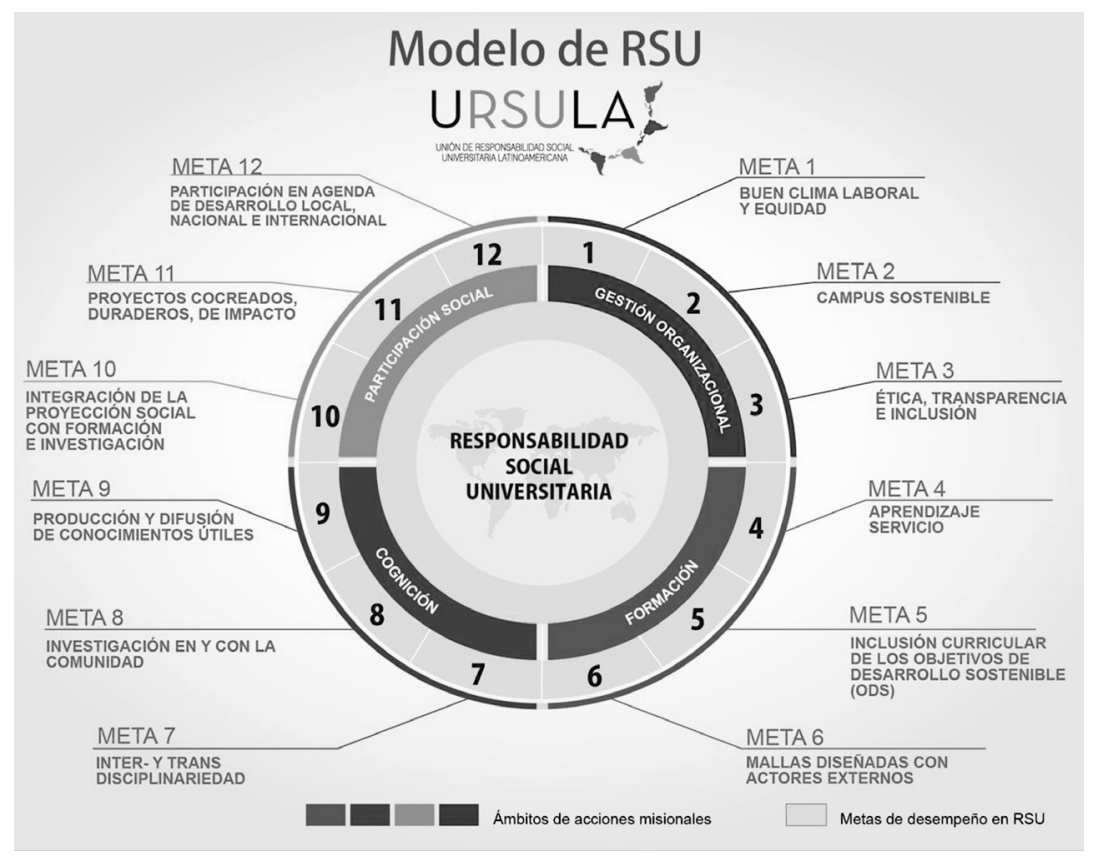

Figura Núm. 1. Modelo URSULA. Fuente: Vallaeys, Solano y Olivera (2019, p.6)

De esta manera, la responsabilidad social universitaria se transforma en la sinergia al interior y exterior de la misma universidad, al interior como un campus responsable, un laboratorio de innovación social para el cumplimiento de los Objetivos del Desarrollo Sustentable; al exterior, como el proceso para la construcción de comunidades de aprendizaje mutuo, de todos los actores involucrados, universitarios y sociedad en general. Actualmente las instituciones de educación superior dentro del área del diseño discurren entre el diseño social y la responsabilidad social universitaria, como su ethos de una "filiación en la verdad" (Guzmán, 2007, p. 138), dentro un mundo cambiante que requiere de la 
participación del diseñador, fusionándose ambos términos como uno solo. Cabe destacar que diseño social y responsabilidad social son términos complementarios uno del otro, pero son independientes en los resultados esperados. La responsabilidad social va más allá del individuo, se dirige al sistema educativo para planificar acciones hacia la formación en este caso, de diseñadores, generando una conciencia y un proceder social, generando con ello, la identidad del diseñador acorde a los tiempos que vive. Sin duda, en cada momento histórico se ha percibido la preocupación de las instituciones de educación superior desde una visión ética que para González es la "razón que funda la esperanza", así el ethos

[...] es esa actitud y acción propias del hombre, por las cuales, sobrepasando la mera naturaleza $[\ldots]$ puede crecer por sí mismo o incendiarse, puede luchar o "venderse"; puede imprimirse así mismo un rostro propio, una manera de ser, que se convierte en su destino. (González, 2007, p. 55)

Desde esta óptica, las instituciones de educación superior se han manifestado como agentes de cambio para enfrentar los desafíos que plantea la dinámica social, política y tecnológica que trae consigo la globalización, así como las transformaciones medio ambientales e impactos sociales que se suceden de manera cotidiana; así han buscado su adaptación a este nuevo y cambiante entorno, atendiendo los requerimientos de la sociedad a través de funciones sustantivas como son

[...] la formación de profesionistas altamente calificados; la generación, la aplicación, la transferencia del conocimiento con un sentido social y la difusión de la cultura y la extensión de los servicios para contribuir al desarrollo nacional y al bienestar de la población. (ANUIES, 2018, p. 79)

En México, el concepto de responsabilidad social ha estado latente en los documentos institucionales de la Asociación Nacional de Universidades e Instituciones de Educación Superior, teniendo como fundamento en un primer momento el bienestar de la población para posteriormente, promover que las instituciones de educación superior asuman el concepto invitándolas a diseñar un modelo hacia tal fin a partir de las pautas que se expresan en la Figura Núm. 2; teniendo como base que toda institución socialmente responsable es la que asegura "que sus funciones se lleven a cabo con la mayor pertinencia, equidad, oportunidad y calidad" (ANUIES, 2018, p. 80). Propuesta que en términos generales concuerda con el Modelo URSULA, al impactar las funciones sustantivas de la docencia, investigación y extensión, en donde se busca acercarse a la realidad del contexto social. 


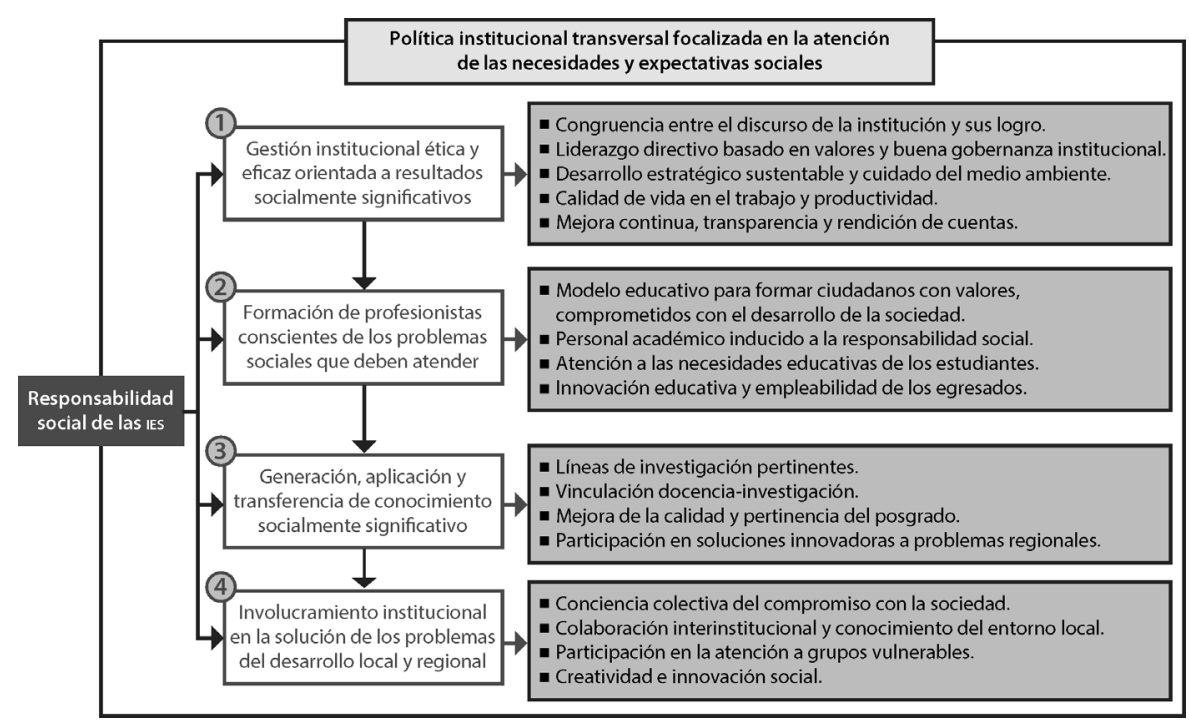

Figura Núm. 2. Mapa conceptual de la responsabilidad social de las IES, desde el enfoque de la Asociación Nacional de Universidades e Instituciones de Educación Superior en México. Fuente: ANUIES (2018, p. 80).

Desde este panorama se plantea la necesidad de determinar la situación que guarda el perfil del diseñador gráfico, con respecto a los principios del paradigma de la responsabilidad social. Recuperando la investigación de Ramírez (2020), en donde se realiza una revisión de los perfiles profesionales inscritos en los planes de estudio Diseño Gráfico en México, se destaca que éste se centra en los conocimientos y habilidades adquiridas durante la formación académica, mencionando que el propósito es formar diseñadores con un enfoque humanista integral, para resolver problemáticas y necesidades a partir del pensamiento crítico. Se destaca la personalidad de emprendedor capaz de ofrecer soluciones que incentiven el dinamismo y el valor de los mercados. Algunas instituciones traspasan el nivel de ejecución de objetos de diseño para un entorno determinado y describen como perfil de egreso la centralidad en el usuario y los factores humanos: profesionistas del diseño y la comunicación, críticos y responsables con su contexto, manteniendo una visión transdisciplinar e intercultural para plantear soluciones creativas a diversos problemas y necesidades de su entorno, tomando al usuario como elemento clave del mensaje, espacio $\mathrm{u}$ objeto de diseño. Existen instituciones que determinan al diseño como una actividad interpretativa y mediadora entre los seres humanos y el entorno, así el diseñador gráfico se concibe como el especialista que tiene como objeto de estudio a la imagen, capaz de designar y gestionar discursos particularmente visuales, por medio de sistemas comunicacionales estratégicos para la intervención de realidades, enfocados hacia la experiencia del usuario, en su beneficio y el de su entorno. 
A partir de esa revisión, se hacer notar que la formación y el perfil de egreso del Diseñador Gráfico aún se encuentran permeados por el enfoque global de las escuelas de negocios, siendo relevante la toma de decisiones desde un pensamiento estratégico, en donde la innovación y el emprendedurismo principalmente, se integran para formar un diseñador que tiene habilidades y capacidades específicas hacia la estética, la utilidad y la comercialización, dentro de un ambiente competitivo; y de manera incipiente se encuentran escuelas que apuestan por el salto hacia el diseño bajo los principios de la responsabilidad social, la cual plantea ámbitos de acción -en este caso formación, cognición y participación socialcon metas e indicadores particulares, que se asocian con la formación del perfil de egreso del futuro diseñador, mismos que se encuentran en la Tabla Núm. 1.

\begin{tabular}{|c|c|c|}
\hline \multicolumn{3}{|c|}{ Ámbitos de acción dentro de la responsabilidad social } \\
\hline Formación & Cognición & Participación Social \\
\hline Aprendizaje Servicio & $\begin{array}{l}\text { Inter y } \\
\text { transdisciplinariedad }\end{array}$ & $\begin{array}{l}\text { Integración de la } \\
\text { proyección social con } \\
\text { formación e } \\
\text { investigación }\end{array}$ \\
\hline $\begin{array}{l}\text { Inclusión curricular de } \\
\text { los } 17 \text { ODS } \\
\text { Mallas diseñadas con } \\
\text { actores externos }\end{array}$ & $\begin{array}{l}\text { Investigación en y con } \\
\text { la comunidad } \\
\text { Producción y difusión } \\
\text { de conocimientos útiles }\end{array}$ & $\begin{array}{l}\text { Proyectos cocreados, } \\
\text { duraderos, de impacto } \\
\text { Participación en agenda } \\
\text { de desarrollo local, } \\
\text { nacional e internacional. }\end{array}$ \\
\hline
\end{tabular}

Tabla Núm. 1. Ámbitos de acción de la RSU, considerados dentro de la Matriz de autodiagnóstico del Modelo URSULA (2019). Fuente: Vallaeys (2019, p.116)

El perfil de egreso del diseñador gráfico desde esta perspectiva, requiere de la participación de la gestión de las instituciones de educación superior para el logro de los objetivos de cada uno de los ámbitos de acción, lo que demuestra que para cambiar el paradigma del ethos hacia una verdadera visión sobre la responsabilidad social, es necesario relevar la estructura, los procesos y la definición de las instituciones dentro del contexto social, en donde es necesario "acostumbrarse a un estado constante desorientación, a viajar por caminos cuya dirección y duración se desconocen, sin mirar más allá de la próxima curva o encrucijada; necesitan concentrar toda su atención en el tramo del camino que deben recorrer antes del anochecer" (Bauman, 2004, pp. 219-220). En este sentido, depende de las instituciones de educación superior responder a los compromisos que tienen como organismos que forman a los futuros profesionistas del diseño.

Tras la conciencia profunda de falsos mundos sublimes y tras la crítica y la crisis radical de valores que ha traído consigo la historia de nuestro tiempo -tanto en el orden de la praxis moral de la cultura en general-, solamente la propia razón crítica puede reencontrar y refundamentar el mundo del sentido y el valor. La crítica de la ética sólo se supera con una ética crítica. (González, 2007, p. 34) 


\section{Conclusiones}

Se reconoce que las entidades encargadas de la evaluación de los programas de estudio han tenido logros con respecto a los estándares de calidad establecidos en cada institución de educación superior evaluada, sin embargo, es necesario analizar si los parámetros a medir permiten de una manera real destacar la calidad y eficiencia de la operatividad de cada uno de los espacios educativos, con sus procesos y resultados obtenidos. O bien, si es posible pensar en otros enfoques para obtener indicadores que ayuden a legitimar la función de las universidades como entidades al servicio de la sociedad.

La identidad del diseñador gráfico del siglo XXI se encuentra en la diyuntiva de su definición para los tiempos en el que vive y sobrevive. La universidad tiene la oportunidad histórica para llegar a sortear los retos que plantea el futuro al ser congruente en el discurso y la praxis en la formación de profesionistas, al establecer una revisión de su función en los tiempos que corren. Los ejes sustantivos de las instituciones de educación superior necesitan integrar, sobre todo, investigación con formación, y esto puede lograrse a partir de generar aprendizajes significativos en consonancia con intervenciones dentro del contexto social. En cierto grado el diseño es una práctica proyectual, pero también es un espacio para el pensamiento, para reflexionar, para promover un desarrollo cognitivo en el estudiante, en cierto modo Papanek (1977) sigue vigente, dado que él afirmaba que para que se logre un mejor diseño, no es necesario enseñar más diseño, sino que el diseñador en ciernes se familiarice con otras áreas del conocimiento, así como desarrollar la conciencia de su responsabilidad moral y social hacia el medio ambiente y así mismo. En este contexto, tal vez conviene ver las posibilidades que plantea la perspectiva de la responsabilidad social universitaria, vista no como una moda o tendencia, sino como el puente para transitar en una sociedad que exige una visión glocal y de un Diseñador Gráfico capaz de identificarse a sí mismo como un ciudadano necesario dentro del conglomerado social, por lo que es, por lo que sabe, y por lo que practica.

Las instituciones de educación superior en la actualidad pueden dar cuenta de la identidad del Diseñador Gráfico posicionado en este siglo XXI, de cómo aprende y cómo recibe el aprendizaje, pero aún falta por abordar la cuestión ética, que incide de manera práctica en el ethos de su pensamiento, de su quehacer cotidiano y forma de ver el mundo. El diseñador debe destacarse por ser un sujeto crítico capaz más allá de dilucidar las necesidades de un usuario, implica generar una conciencia de la realidad de sí mismo y de los semejantes, para relacionarse con sujetos y no objetos, desde una visión ética que promueva la sociabilización del conocimiento que en términos llanos, es la base de la responsabilidad social que buscan las instituciones educativas de educación superior.

Desde esta participación se vislumbran diversas líneas de investigación desde el Diseño Gráfico, a partir de su concepción y revitalización como programa de estudio, así como la necesaria intervención del diseñador dentro del conglomerado social; asimismo, cuál es el ethos que permea a las instituciones de educación superior y cuál es el ethos deseable desde la responsabilidad social y cuáles son las estrategias para lograrlo, las pautas están definidas desde los modelos revisados. Procurar esta reflexión permitirá analizar su permanencia o transformación del diseño y del diseñador, así como de las instituciones de 
educación superior desde el cuestionamiento de lo que implica ser responsable desde la dimensión humana, en el contexto local y global, sin perder de vista como lo mencionó en su momento Morin.

"el hombre ha creado nuevas esferas de vida: la vida del espíritu, la vida de los mitos, la vida de las ideas, la vida de la conciencia [...] A partir y más allá de sus identidades que lo enraizan en la tierra y se inscriben en el cosmos, el hombre produce sus identidades propiamente humanas..." (Morin, 2006, p. 61)

\section{Fuentes de información}

Asociación Nacional de Universidades e Instituciones de Educación Superior. (2018). Visión y acción 2030. Propuesta de la ANUIES para renovar la educación superior en México. México: ANUIES

Bauman, Z. (2004). Modernidad Líquida. Argentina: FCE

Beck, U. (2008). ¿Qué es la globalización? Falacias del globalismo, respuestas a la globalización. España: Paidós.

Belluccia, R. (2007). El diseño gráfico y su enseñanza. Ilusiones y desengaños. Argentina: Paidós, Estudios de Comunicación.

Comaprod. (2017). Marco de referencia e Instrumento de Acreditación. México: Consejo Mexicano para la acreditación de programas de diseño A.C.

Comaprod. (2020). Nosotros COMAPROD. Recuperado de http://www.comaprod.com/ nosotros/

Deval, J. (2004). Los fines de la educación. México: Siglo XXI editores.

Didou, S. (2015). Responsabilidad Social Universitaria en América Latina: Recursos y Controversias. En Aponte, E. (ed.) (2015). La Responsabilidad Social de las Universidades: Implicaciones para América Latina y el Caribe. Puerto Rico: UNESCO-IESALC

Fernández Fassnacht, Enrique. (2017). Una mirada a los desafíos de la educación superior en México. Innovación educativa (México, DF), 17(74), 183-207. Recuperado de http:// www.scielo.org.mx/scielo.php?script=sci_arttext\&pid=S1665-26732017000200183\&ln $\mathrm{g}=\mathrm{es} \& \mathrm{t} \operatorname{lng}=\mathrm{es}$.

Frascara, J. (1994). Diseño gráfico y comunicación. Argentina: Ediciones Infinito.

Gónzales, J. (2007). Ética y Libertad. México. FCE, FFL,UNAM.

Gualdrón, N. (2017). El diseño para el bienestar humano. El sentido y contenido del discurso gráfico. Revista Luciérnaga - Comunicación. Facultad de Comunicación Audiovisual Politécnico Colombiano Jaime Isaza Cadavid \& Facultad de Ciencias de la Comunicación - Universidad Autónoma de San Luis Potosí - México. DOI: 10.33571/revistaluciernaga Guzmán, D. (2007). El ethos filosófico. Praxis Filosófica. Nueva serie, No. 24, Enero-Junio 2007: 137-145 ISSN: 0120-4688. Recuperado de: https://mansunides.org/es/rsc/responsabilidad-social-corporativa/historia-rsc 
Mans Unides. ONG contra la pobreza en el mundo. Historia de la RSE.

Margolin, V. y Margolin, S. (2012). Un "modelo social" de diseño: cuestiones prácticas e investigación. Revista KEPES Año 9 No. 8 enero-diciembre 2012, págs. 61-71 ISSN 1794-7111 Recuperado de http://vip.ucaldas.edu.co/kepes/downloads/Revista8_4.pdf

Morin, E. (2006). Tierra-patria. Buenos Aires: Nueva Visión.

Padierna, M.P. (2016). La «sociedad del conocimiento» y el campo de las políticas de transformación de la educación superior. Reseña. resu.anuies.mx Revista de la Educación Superior 45(179) (2016) 117-120 R

Papanek, V. (1977). Diseñar para el mundo real. Ecología humana y cambio social. España: Editorial Blume.

Pérez, A.I. (2004). La cultura escolar en la sociedad neoliberal. España: ediciones Morata.

Polo, M.A. (2013). Pâthos, êthos y vida atenta. Revista Horizonte de la Ciencia. Horizonte de la Ciencia 3 (4), julio 2013. FE-UNCP/ISNN 2304 - 4330.

Ramírez, B. (2020). Estructuración de un programa de especialización enfocado al desarrollo del diseñador gráfico como emprendedor y gestor empresarial. Tesis de grado de la Maestría en Diseño. Universidad Autónoma del Estado de México.

Rivera, L. (2007). La retórica en el diseño gráfico. México: Encuadre, Asociación Mexicana de Escuelas de Diseño Gráfico.

Rodríguez, L. (2010). El diseño en la Posmodernidad. Discursos y tesis. Legado De Arquitectura Y Diseño, 5(7), 49-62. Recuperado de https://legadodearquitecturaydiseno. uaemex.mx/article/view/13926

Rousseau, J. (2000). Emilio o La educación. Elaleph.com. Recuperado de http://www.educ.ar

Salas, I. Et.Al. (2019). La apropiación de la política educativa en las universidades públicas mexicanas: entre la ambigüedad y la diversidad. Resu.anuies.mx Revista de la Educación Superior 48 (191) (2019). Recuperado de http://creativecommons.org/licenses/by-nc-nd/4.0/

Savater, F. (2003). El valor de educar. México: Ariel.

Subirat, J. y Badosa, J. (2008). ¿Qué diseño para qué sociedad?. Notas sobre la funcionalidad social del diseño. Recuperado de https://www.academia.edu/23667186/_Qu\%C3\%A9_ dise\%C3\%B1o_para_qu\%C3\%A9_sociedad_._Notas_sobre_la_funcionalidad_social_del_dise\%C3\%B1o

Tapia, A. (2005a). El diseño gráfico en el espacio social. México: Designio. Teoría y práctica Tapia, A. (2005b). Hacia una definición del diseño gráfico. Asociación Mexicana de Escuelas de Diseño Gráfico. https://encuadre.org/hacia-una-definicion-del-diseno-grafico/

Tomassino, H., y Cano, A. (2016). Modelos de extensión universitaria en las universidades latinoamericanas en el siglo XXI: tendencias y controversias. Universidades, (67),7-24. ISSN: 0041-8935. Recuperado de https://www.redalyc.org/articulo.oa?id=373/37344015003

Torres, I. (2014). Diseño crítico: de la transgresión a la autonomía. Proyecto de grado. Magister Universitario en Investigación en Arte y Diseño. Barcelona: Universidad de Barcelona. Recuperado de https://ddd.uab.cat/pub/trerecpro/2015/hdl_2072_255203/2014_2015_torres_fernandez_inmaculada.pdf

Vallaeys, F. (2014). La responsabilidad social universitaria: un nuevo modelo universitario contra la mercantilización. Revista iberoamericana de educación superior, 5(12), 105-117. Recuperado de http://www.scielo.org.mx/scielo.php?script=sci_arttext\&pid=S200728722014000100006\&lng=es\&tlng=es. 
Vallaeys, F. (2016). Introducción a la Responsabilidad Social Universitaria (RSU). Colección Responsabilidad Social Empresarial y Universitaria. Colombia: Ediciones Universidad Simón Bolivar. ISBN 978-958-8930-65-7. Recuperado de http://unionursula.org/wpcontent/uploads/2016/11/Libro-intrpduccion-a-la-rsu-francois-vallaeys.pdf

Vallaeys, F. (2019). Responsabilidad Social Universitaria, El modelo URSULA, Estrategias, herramientas, indicadores. Perú. Unión de Responsabilidad Social Universitaria Latinoamérica. (URSULA). Recuperado de http://unionursula.org/wp-content/uploads/2019/12/ ursula-modelo-responsabilidad-social-universitaria-rsu.pdf

Vallaeys, F. y Álvarez, J. (2019). Hacia una definición latinoamericana de responsabilidad social universitaria. Aproximación a las preferencias conceptuales de los universitarios. Educación XX1, 22(1), 93-116, doi: 10.5944/educXX1.19442

Vallaeys, F., Solano, D. y Olivera, M. (2019). 2a . Investigación Continental sobre el estado del arte de la Responsabilidad Social Universitaria en América latina. [presentación ejecutiva Modelo URSULA]. Recuperado el 21 junio 2020, de http://unionursula.org/wp-content/ uploads/2019/04/presentacion-i-c-rsu-ursula-2019-espanol.pdf

Zárate, R. y García, S. (2014). La cultura socialmente responsable de la UIS: una perspectiva desde el ámbito educativo y social. Revista Encuentros. Universidad Autónoma del Caribe, 12 (2), pp. 105-120. Recuperado de https://www.researchgate.net/publication/287562248_La_cultura_socialmente_responsable_de_la_UIS_una_perspectiva_desde_el_ambito_educativo_y_social

Zúñiga, J. (2003). Ivan Illich: hacia una desescolarización. Revista Educare No. IV. (47-60). Recuperado de https://www.researchgate.net/publication/279419371_Ivan_Illich_hacia_una_desescolarizacion

\begin{abstract}
Currently the Higher Education Institutions (HEIs) are immersed in a constant dialectical game between the responsibility that it has as a training institution for future professionals against the expectations of society. The educational field of Design is not the exception; Future designers are immersed in the inertia promoted by universities through their study programs, highlighting the relationship that HEIs pronounce with the social context, in that way, future professionals are expected to respond to the society needs; nevertheless, this premise is diluted by appearing as a plausible, but not real, discourse and is described only as a resource to legitimize the efforts of the universities within the Global Society; Thus, this participation has the objective of analyzing the identity of the designer, translated as the professional profile, an indicator that evaluates the effectiveness of the educational process itself, the resource to challenge a workplace, where the mission of HEIs He confronts himself and the identity of the graduate is far from the principles of University Social Responsibility (USW), which seeks recognition of himself and the other, for a better society.
\end{abstract}

Keywords: Identity - Design - Designer - Profile - University 
Resumo: Atualmente, as Instituições de Educação Superior (IES) se encontram interessadas em um constante jogo dialético entre a responsabilidade que tem uma instituição formadora de futuros profissionais e as expectativas da sociedade. No âmbito educativo dos designers gráficos não é exceção. Os futuros designers gráficos se encontram imersos na inércia que promovem as universidades por meio de seus programas de estudos, onde se destaca a relação pronunciada pelas IES com o contexto social. Ou seja, espera que os futuros profissionais ofereçam respostas às necessidades da sociedade. No entanto, essa premissa se dissolve ao figurar-se como um discurso aceitável, porém, não real, e se descreve somente como um recurso para legitimar os esforços das universidades dentro da sociedade global. Assim, este artigo, tem como objetivo analisar a identidade do profissional de design gráfico, traduzida como o perfil profissional indicador que avalia a eficácia do processo educativo, e, é em si mesmo, o recurso para desafiar o local de trabalho, onde a missão das IES se confronta com a identidade do graduando, que por sua vez, está bem distante dos princípios de Responsabilidade Social Universitária (RSU), que busca o reconhecimento de si e do outro, para uma sociedade melhor.

Palavras chave: Identidade - Desenho - Designer - perfil - universidade.

[Las traducciones de los abstracts fueron supervisadas por el autor de cada artículo] 